\title{
Uncovering Perceived Identification Accuracy of In-Vehicle Biometric Sensing
}

Abdallah El Ali

Centrum Wiskunde \&

Informatica

Amsterdam, The Netherlands

abdallah.el.ali@cwi.nl

Liam Ashby

Centrum Wiskunde \&

Informatica

Faculty of Social and

Behavioural Sciences

University of Amsterdam

Amsterdam, The Netherlands

ashbyliam40@gmail.com

\section{Andrew M. Webb}

Centrum Wiskunde \& Informatica

Amsterdam, The Netherlands

amwebb@acm.org
Robert Zwitser

Faculty of Social and

Behavioural Sciences

University of Amsterdam

Amsterdam, The Netherlands

r.j.zwitser@uva.nl

\section{Pablo Cesar}

Centrum Wiskunde \& Informatica

Amsterdam, The Netherlands

p.s.cesar@cwi.nl

Permission to make digital or hard copies of part or all of this work for personal or classroom use is granted without fee provided that copies are not made or distributed for profit or commercial advantage and that copies bear this notice and the full citation on the first page. Copyrights for third-party components of this work must be honored. For all other uses, contact the Owner/Author.

AutomotiveUI '19 Adjunct, September 21-25, 2019, Utrecht, Netherlands

(02019 Copyright is held by the owner/author(s).

ACM ISBN 978-1-4503-6920-6/19/09.

https://doi.org/10.1145/3349263.3351506

\begin{abstract}
Biometric techniques can help make vehicles safer to drive, authenticate users, and provide personalized in-car experiences. However, it is unclear to what extent users are willing to trade their personal biometric data for such benefits. In this early work, we conducted an open card sorting study $(\mathrm{N}=11)$ to better understand how well users perceive their physical, behavioral and physiological features can personally identify them. Findings showed that on average participants clustered features into six groups, and helped us revise ambiguous cards and better understand users' clustering. These findings provide the basis for a follow up online closed card sorting study to more fully understand perceived identification accuracy of (in-vehicle) biometric sensing. By uncovering this at a larger scale, we can then further study the privacy and user experience trade-off in (automated) vehicles.
\end{abstract}

\section{Author Keywords}

Biometrics; sensing; card sorting; perceived accuracy; privacy; in-vehicle

\section{CCS Concepts}

-Human-centered computing $\rightarrow \mathrm{HCl}$ theory, concepts and models; 


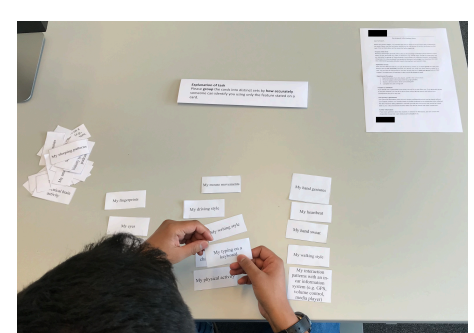

Figure 1: A participant sorting cards.

\section{Introduction}

Biometric techniques can make vehicles safer to drive (e.g. drowsiness detection [44]), protect them against theft (e.g., tap-based authentication [20]), provide higher cost efficiency (e.g., rewarding good driver profiles through insurance telematics [11]), or provide personalized in-car experiences (e.g., route personalization based on driving style [6]). These advantages and benefits notwithstanding, biometric techniques and the promise of connected cars also raise large privacy concerns [6, 45], for example concerning the privacy ${ }^{1}$ and security of (sensed) personal data [35].

Prior research has addressed these issues by focusing on the so-called privacy-personalization paradox, namely that consumers who value information transparency are also less likely to participate in personalization [2]. This phenomenon has been generally less studied in an automotive context, and for automated driving in particular. The latter context becomes especially relevant, where non-drivingrelated activities (e.g., texting, eating) are desirable to perform in the car [34], which brings about opportunities for a wider range of in-vehicle biometric sensing [36]. To address the question of trading personal biometric data for in-vehicle user benefits, we take the first step here using an open card sorting study to better understand how well users perceive their physical, behavioral and physiological features can personally identify them. We contribute early empirical findings of how participants interpret and cluster such features.

\section{Background and Related work}

According to Jain et al. [15], biometric recognition "can be defined as the science of establishing the identity of an individual based on the physical and/or behavioral characteristics of the person either in a fully automated or a semiautomated manner." Furthermore, the context in which data

\footnotetext{
${ }^{1}$ See also EU GDPR on vehicles: http://tiny.cc/7abj8y; 19.06.2019
}

is collected, and who the data is to be sold to (e.g. research institute, commercial company), have been found to impact users' willingness to disclose personal information [38]. Past research has also investigated how different types of personal information are valued differently by users. For example, amongst various types of data (i.e. application, location, communication), location data has been found to be the most valuable to users [40].

Within the automotive domain, there has been prior work on automotive activity recognition, for example using capacitive proximity sensing [4], that does not rely on collecting highly personal user data. Other work addresses the privacy implications of accelerometer data, where geographic tracking of drivers becomes possible using only a phone's accelerometer sensor [18]. However, there is still no systematic analysis of how and when drivers and passengers willing to share their personal, biometric data, in exchange for a better in-vehicle trip experience.

\section{Methods}

Open card sorting

Open card sorting is a widely-used method (in web design) to create taxonomies based on users' groupings of the content. Importantly, it can be effective for discovering the optimal organization of information according to users' viewpoints [48]. Furthermore, it has been shown to have high cross-study reliability [16]. Since we are concerned with biometric technologies and perceived identification accuracy, card sorting is a suitable method to better understand how users cluster physical, behavioral and physiological features that such technologies can sense.

\section{Card selection process}

To understand how users perceive different biometric features, we first collect a common, relevant set of features, 
AutomotiveUI '19 Adjunct, September 21-25, 2019, Utrecht, Netherlands

\begin{tabular}{|c|c|c|c|c|c|c|c|}
\hline \# & Card & Example Technology & Ref & \# & Card & Example Technology & Ref \\
\hline 1 & My face & Face recognition $(2 \mathrm{D}, 3 \mathrm{D})$ & {$[31,35]$} & 21 & My posture & Posture recognition & [33] \\
\hline 2 & My facial expressions & Facial emotion expression recognition & {$[31,5]$} & 22 & My media listening history & Personalized music emotion recognition & [49] \\
\hline 3 & My ears & Ear identification & [25] & 23 & My media watching history & Profiling TV viewers using data mining & [39] \\
\hline 4 & My eyes & Eye tracking; iris and retina recognition & [31] & 24 & My driving style & Driver and driving style recognition & [43] \\
\hline 5 & My physical activity & $\begin{array}{l}\text { Physical activity recognition (ac- } \\
\text { celerometers) }\end{array}$ & [24] & 25 & $\begin{array}{l}\text { My interaction patterns with an } \\
\text { in-car information system }\end{array}$ & Modelling driver IVIS interactions & [12] \\
\hline 6 & My fingerprints & Fingerprint recognition & {$[31,35]$} & 26 & My SMS messages & $\begin{array}{l}\text { User classification based on SMS mes- } \\
\text { sages }\end{array}$ & [14] \\
\hline 7 & My walking style & Gait recognition & [31] & 27 & My locations on a given day & Location tracking & [7] \\
\hline 8 & My hands & $\begin{array}{l}\text { Hand geometry recognition }(2 \mathrm{D}, 3 \mathrm{D}) \text {; } \\
\text { Palm and finger vein recognition }\end{array}$ & {$[31,35,37]$} & 28 & My hand sweat & $\begin{array}{l}\text { Galvanic Skin Response for emotion } \\
\text { recognition }\end{array}$ & [23] \\
\hline 9 & My sleeping patterns & Sleep classification & [21] & 29 & My hand gestures & Hand gesture recognition & [32] \\
\hline 10 & My smell & Odor recognition & [31] & 30 & My electrical brain activity & Electroencephalography; fNIRS & {$[41,36]$} \\
\hline 11 & My handwriting & $\begin{array}{l}\text { Optical Character Recognition (OCR); } \\
\text { signature recognition }\end{array}$ & {$[26,31,8]$} & 31 & My breathing & Breathing monitoring & [30] \\
\hline 12 & $\begin{array}{l}\text { My touches on a smartphone } \\
\text { (e.g., movement, pressure, etc.) }\end{array}$ & Touchscreen dynamics & [31] & 32 & My genetic makeup & DNA matching & [31] \\
\hline 13 & My mouse movements & Mouse movement dynamics & [31] & 33 & My muscle movements & Electromyography motion classification & [29] \\
\hline 14 & My typing on a keyboard & Keystroke recognition & [31] & 34 & My personality & Personality classification & \\
\hline 15 & My voice & $\begin{array}{l}\text { Speaker identification, verification, } \\
\text { authentication }\end{array}$ & {$[10,35]$} & 35 & My eye gaze patterns & Eye tracking and user identification & {$[31,13]$} \\
\hline 16 & My teeth & Teeth recognition & [19] & 36 & My driving route & Personalized route recommendation & [22] \\
\hline 17 & My footprints & Footprint (size and shape) recognition & [28] & 37 & My company in a vehicle & $\begin{array}{l}\text { Person identification using social con- } \\
\text { nections }\end{array}$ & {$[47,46]$} \\
\hline 18 & My heartbeat & $\begin{array}{l}\text { Electrocardiogram - Heart Rate Vari- } \\
\text { ability }\end{array}$ & [31] & 38 & My body temperature & Thermal imaging & [1] \\
\hline 19 & My writing style & Stylometry & [31] & 39 & My eating style & $\begin{array}{l}\text { Eating episode classification (wearable } \\
\text { sensors) }\end{array}$ & [3] \\
\hline 20 & My smartphone app usage & App usage fingerprints & [42] & 40 & My saliva & Stress (from cortisol) recognition & [17] \\
\hline
\end{tabular}

Table 1: Cards (features), example biometric technology, and citation. For this study, we only tested cards 1-32 (plus "My signature").

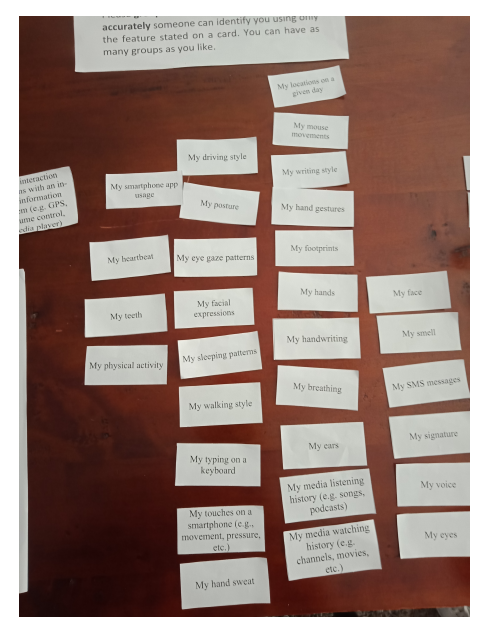

Figure 2: Set of sorted cards by a participant. framed in an understandable manner. This means deliberately not mentioning the underlying biometric technology, and instead only presenting the physical, behavioral or physiological feature that a technology processes. For example, to investigate facial recognition, we present participants with the card "My face". Within an (automated) driving context, the space of biometrics is quite vast [36]. We did an extensive literature search by querying the ACM Digital Library and Google Scholar for papers published on biometric techniques, across all contexts (automotive, mobile, health, etc.). We then selected any biometrics that could in principle be also used in an (automated) vehicle. Our initial search resulted in 33 cards, which we test in this study. Our ongoing search resulted in eight more features (33-40) $)^{2}$, which we aim to test in a follow up study. All cards are shown in Table 1. While not exhaustive, this list is sufficient for investigating perceptions of common biometrics.

\footnotetext{
2"My signature" was discarded, as explained later
}

\section{Procedure}

Participants were tested in a lab environment, They were provided with an information sheet, filled and signed a consent and participant information form, then given a task demonstration. They were provided with sorting instructions on the desk (Figure 1). Instructions stated: "Please group the cards into distinct sets by how accurately someone can identify you using only the feature stated on a card." We deliberately did not constrain participants to consider they were inside a vehicle for two reasons: (a) to gain a general understanding of perceived identification accuracy (b) to not burden participants to reflect on both automated and non-automated driving. After they sorted the cards, they were asked to label each group. There was no time limit, nor a limit to the number of groups they can create. Participants were encouraged to think aloud, and asked to explain their final groupings. Each session was audio recorded, and lasted approximately 10-30 min. 


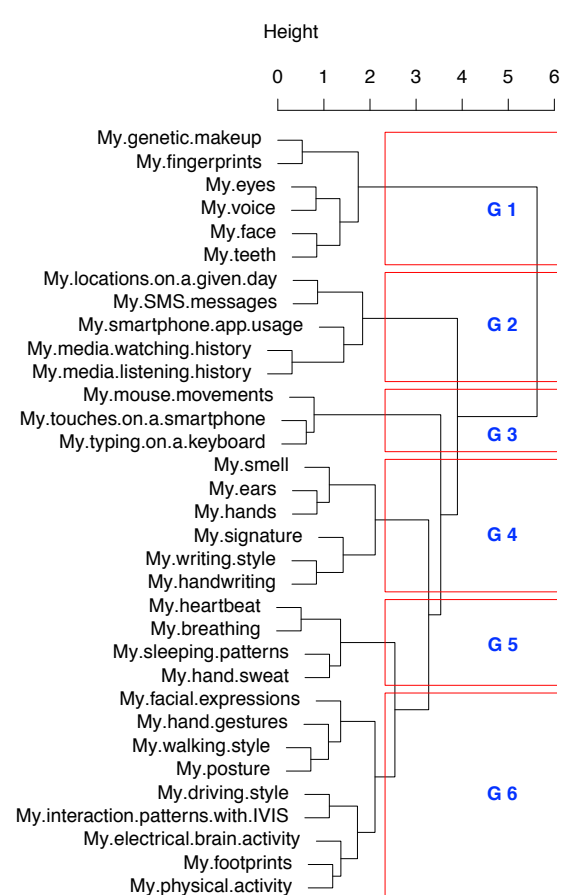

Figure 3: Dendrogram $(k=6)$.

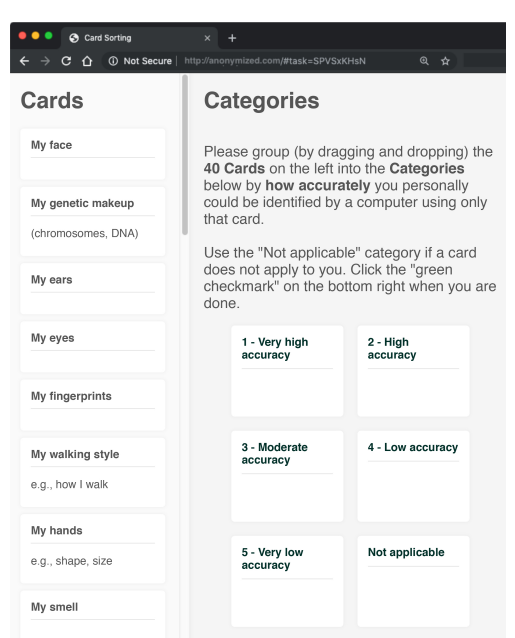

Figure 4: Screenshot of the closed card sorting website.

\section{Participants}

We recruited 11 participants $(6 f, 5 \mathrm{~m})$, aged between 21-38 $(M=25.6, S D=5.4)^{3}$. Eight were students, and the remainder graduate-level or higher. Seven stated they had a technical background. Three participants had driving experience, two were learning how to drive, and the rest no experience. Participants did not receive monetary compensation.

\section{Early Results and Discussion}

Grouping, labeling, and task instructions

The mean group size created was $5.8(\mathrm{SD}=1.1)$. Given the question we asked, participants largely created a ranked list of groups, ranging from high (e.g., P2: "That's me!"; P1: "Bio features") to low perceived identification accuracy (P9: "Things I do not or do almost the same as other people"; P5: "No one can identify me (not enough info)"). Overall, participants' labels were consistent, however differed by grouping size. Given the range and mean group size, we aim to test six categories for our future study.

To better understand how our cards were grouped, we used Ward's hierarchical agglomerative clustering method [27] with $\mathrm{k}=6$ clusters (creating six groups: G1-G6), where the resulting dendrogram using Jaccard similarity is shown in Figure 3 . The dendrogram shows that some while groups contain physical features (e.g., G1: genetic makeup, fingerprints, eyes, ...), others are geared towards interactions with technology (e.g., G3: mouse movements, touches on smartphone, ...). The last grouping (G6) contains inconsistent topics, where some are quite identifiable characteristics of individuals (e.g., facial expressions), whereas others less so (e.g., driving style). This leads us to consider adding a "Not applicable" (N/A) category. Finally, one participant raised the question of whether a person or machine is doing

\footnotetext{
${ }^{3}$ Age statistics are based on 10 participants only, as one participant chose not to disclose their age.
}

the identification. Given this, we will adjust our future instructions to reflect a focus on machine (computer) sensing.

\section{Ambiguous and redundant cards}

Not all the cards were immediately understandable to participants (e.g., P2 was confused by physical activity and genetic makeup). Due to this, we need to provide additional examples (beyond what is listed in Table 1) to decrease ambiguity. P3 was unsure why we have cards for "My face" and "My facial expressions" - while this may create redundancy, we find it is better to maintain separation with respect to the underlying recognition technology. Moreover, cultural factors were surfaced, for example concerning writing style (P2: "In Chinese I have a very special writing style...when people see my homework, they know it is me"). We also merged "My signature" with "My handwriting", which was found to be redundant. Finally, some cards were deemed inapplicable, e.g., if participants did not have driving experience (P9: "I can't drive...kind of just eliminated that") - this further necessitates an N/A category.

\section{Next Steps}

Our open card sort study findings helped shape a follow up closed card sorting study, where we have built our own online tool to collect closed card sort data (Figure 4). We have modified our instructions and the number of closed categories according to insights gathered. We also now in clude the complete 40 card list, and provide examples for most cards to avoid ambiguity. Based on the closed card sort data, we will choose the extreme ends of identifiability categories, and use those for a follow up study on identifying (automated) in-vehicle user experience privacy tradeoffs (e.g., trading heartbeat data for alertness monitoring). 


\section{REFERENCES}

1. Yomna Abdelrahman, Eduardo Velloso, Tilman Dingler, Albrecht Schmidt, and Frank Vetere. 2017. Cognitive Heat: Exploring the Usage of Thermal Imaging to Unobtrusively Estimate Cognitive Load. Proc. ACM Interact. Mob. Wearable Ubiquitous Technol. 1, 3, Article 33 (Sept. 2017), 20 pages. DOI : http://dx.doi.org/10.1145/3130898

2. Naveen Farag Awad and M. S. Krishnan. 2006. The Personalization Privacy Paradox: An Empirical Evaluation of Information Transparency and the Willingness to Be Profiled Online for Personalization. MIS Quarterly 30, 1 (2006), 13-28.

http://www.jstor.org/stable/25148715

3. Abdelkareem Bedri, Richard Li, Malcolm Haynes, Raj Prateek Kosaraju, Ishaan Grover, Temiloluwa Prioleau, Min Yan Beh, Mayank Goel, Thad Starner, and Gregory Abowd. 2017. EarBit: Using Wearable Sensors to Detect Eating Episodes in Unconstrained Environments. Proc. ACM Interact. Mob. Wearable Ubiquitous Technol. 1, 3, Article 37 (Sept. 2017), 20 pages. DOI : http://dx. doi .org/10.1145/3130902

4. Andreas Braun, Sebastian Frank, Martin Majewski, and Xiaofeng Wang. 2015. CapSeat: Capacitive Proximity Sensing for Automotive Activity Recognition. In Proc. AutomotiveUl '15. ACM, New York, NY, USA, 225-232. DOI : http://dx.doi.org/10.1145/2799250.2799263

5. Carlos Busso, Zhigang Deng, Serdar Yildirim, Murtaza Bulut, Chul Min Lee, Abe Kazemzadeh, Sungbok Lee, Ulrich Neumann, and Shrikanth Narayanan. 2004. Analysis of Emotion Recognition Using Facial Expressions, Speech and Multimodal Information. In Proc. ICMI '04. ACM, New York, NY, USA, 205-211. DOI : http://dx.doi.org/10.1145/1027933.1027968
6. Riccardo Coppola and Maurizio Morisio. 2016. Connected Car: Technologies, Issues, Future Trends. ACM Comput. Surv. 49, 3, Article 46 (Oct. 2016), 36 pages. DOI: http://dx.doi.org/10.1145/2971482

7. Yves-Alexandre de Montjoye, Hidalgo Cesar A, Michel Verleysen, and Vincent D Blondel. 2013. Unique in the Crowd: The privacy bounds of human mobility. Scientific Reports 3 (2013).

8. P. Dhayarkar and R. Itkarkar. 2016. Comparison analysis for signature verification of bank cheque. In 2016 International Conference on Automatic Control and Dynamic Optimization Techniques (ICACDOT). 906-909. DOI :

http://dx.doi.org/10.1109/ICACDOT.2016.7877718

9. Sheng Fang, Catherine Achard, and Séverine Dubuisson. 2016. Personality Classification and Behaviour Interpretation: An Approach Based on Feature Categories. In Proc. ICMI '16. ACM, New York, NY, USA, 225-232. DOI :

http://dx.doi.org/10.1145/2993148.2993201

10. Chuhan Gao, Varun Chandrasekaran, Kassem Fawaz, and Suman Banerjee. 2018. Traversing the Quagmire That is Privacy in Your Smart Home. In Proceedings of the 2018 Workshop on loT Security and Privacy (IoT S\&\#38;P '18). ACM, New York, NY, USA, 22-28. DOI : http://dx.doi.org/10.1145/3229565.3229573

11. P. Handel, I. Skog, J. Wahlstrom, F. Bonawiede, R. Welch, J. Ohlsson, and M. Ohlsson. 2014. Insurance Telematics: Opportunities and Challenges with the Smartphone Solution. IEEE Intelligent Transportation Systems Magazine 6, 4 (winter 2014), 57-70. DOI : http://dx.doi.org/10.1109/MITS.2014.2343262 
12. C. Harvey. 2011. Modelling and evaluating drivers? interactions with in-vehicle information systems (IVIS). Ph.D. Dissertation. University of Southampton. https://eprints.soton.ac.uk/348898/

13. C. Holland and O. V. Komogortsev. 2011. Biometric identification via eye movement scanpaths in reading In 2011 International Joint Conference on Biometrics (IJCB). 1-8. DOI :

http://dx.doi.org/10.1109/IJCB. 2011.6117536

14. D. Hu, F. Sun, L. Tu, and B. Huang. 2013. We Know What You Are-A User Classification Based on Mobile Data. In 2013 IEEE International Conference on Green Computing and Communications and IEEE Internet of Things and IEEE Cyber, Physical and Social Computing. 1282-1289. DOI : http://dx.doi.org/10. 1109/GreenCom-iThings-CPSCom. 2013.223

15. Anil K. Jain, Arun A. Ross, and Karthik Nandakumar. 2011. Introduction to Biometrics. Springer Publishing Company, Incorporated.

16. Christos Katsanos, Nikolaos Tselios, Nikolaos Avouris, Stavros Demetriadis, Ioannis Stamelos, and Lefteris Angelis. 2019. Cross-study Reliability of the Open Card Sorting Method. In Extended Abstracts of the $2019 \mathrm{CHI}$ Conference on Human Factors in Computing Systems (CHI EA '19). ACM, New York, NY, USA, Article LBW2718, 6 pages. DOI :

http://dx.doi.org/10.1145/3290607.3312999

17. Tara Kidd, Livia A. Carvalho, and Andrew Steptoe. 2014. The relationship between cortisol responses to laboratory stress and cortisol profiles in daily life. Biological Psychology 99 (2014), 34 - 40. DOI : http://dx.doi.org/https: //doi.org/10.1016/j.biopsycho.2014.02.010
18. Jacob Leon Kröger, Philip Raschke, and Towhidur Rahman Bhuiyan. 2019. Privacy Implications of Accelerometer Data: A Review of Possible Inferences. In Proceedings of the 3rd International Conference on Cryptography, Security and Privacy (ICCSP '19). ACM, New York, NY, USA, 81-87. DOI : http://dx.doi.org/10.1145/3309074.3309076

19. R. Kumar. 2016. Teeth recognition for person identification. In 2016 International Conference on Computation System and Information Technology for Sustainable Solutions (CSITSS). 13-16. DOI : http://dx.doi.org/10.1109/CSITSS. 2016.7779432

20. Andrew L. Kun, Travis Royer, and Adam Leone. 2013. Using Tap Sequences to Authenticate Drivers. In Proc. AutomotiveUl '13. ACM, New York, NY, USA, 228-231. DOI : http://dx.doi .org/10.1145/2516540.2516567

21. Martin Längkvist, Lars Karlsson, and Amy Loutfi. 2012. Sleep Stage Classification Using Unsupervised Feature Learning. Adv. Artif. Neu. Sys. 2012, Article 5 (Jan. 2012), 1 pages. DOI : http://dx.doi.org/10.1155/2012/107046

22. Long Liu, Jin Xu, Stephen Shaoyi Liao, and Huaping Chen. 2014. A Real-time Personalized Route Recommendation System for Self-drive Tourists Based on Vehicle to Vehicle Communication. Expert Syst. Appl. 41, 7 (June 2014), 3409-3417. DOI : http://dx.doi.org/10.1016/j.eswa. 2013.11.035

23. M. Liu, D. Fan, X. Zhang, and X. Gong. 2016. Human Emotion Recognition Based on Galvanic Skin Response Signal Feature Selection and SVM. In Proc. ICSCSE '16. 157-160. DOI : http://dx.doi.org/10.1109/ICSCSE. 2016.0051 
24. Yonggang Lu, Ye Wei, Li Liu, Jun Zhong, Letian Sun, and Ye Liu. 2017. Towards unsupervised physical activity recognition using smartphone accelerometers. Multimedia Tools and Applications 76, 8 (01 Apr 2017), 10701-10719. DOI :

http://dx.doi.org/10.1007/s11042-015-3188-y

25. Rahim Mohd Shafry Mohd, Amjad Rehman, Fajri Kurniawan, and Tanzila Saba. 2017. Ear biometrics for human classification based on region features mining. Biomedical Research (0970-938X) 28, 10 (2017), 4660 - 4664. http://search.ebscohost.com/login. aspx? direct $=$ true\&db=as $\& A N=123606315 \&$ site $=$ eds $-l$ ive

26. Shunji Mori, Hirobumi Nishida, and Hiromitsu Yamada. 1999. Optical Character Recognition (1st ed.). John Wiley \& Sons, Inc., New York, NY, USA.

27. Fionn Murtagh and Pierre Legendre. 2014. Ward's Hierarchical Agglomerative Clustering Method: Which Algorithms Implement Ward's Criterion? J. Classif. 31, 3 (Oct. 2014), 274-295. DOI : http://dx.doi.org/10.1007/s00357-014-9161-z

28. Kapil Kumar Nagwanshi and Sipi Dubey. 2018.

Statistical Feature Analysis of Human Footprint for Personal Identification Using BigML and IBM Watson

Analytics. Arabian Journal for Science and Engineering 43, 6 (01 Jun 2018), 2703-2712. DOI :

http://dx.doi.org/10.1007/s13369-017-2711-z

29. Nurhazimah Nazmi, Mohd Azizi Abdul Rahman, Shinichirou Yamamoto, Siti Anom Ahmad, Hair Zamzuri, and Saiful Amri Mazlan. 2016. A review of classification techniques of EMG signals during isotonic and isometric contractions. Sensors 16, 8 (17 8 2016). DOI : http: //dx. doi .org/10.3390/s16081304

30. Jinglong Niu, Maolin Cai, Yan Shi, Shuai Ren, Weiqing Xu, Wei Gao, Zujin Luo, and Joseph Reinhardt. 2019.
A Novel Method for Automatic Identification of Breathing State. Scientific Reports 9 (12 2019). DOI : http://dx.doi . org/10.1038/s41598-018-36454-5

31. M.S. Obaidat, I. Traore, and I. Woungang. 2018. Biometric-Based Physical and Cybersecurity Systems. Springer International Publishing.

https://books.google.nl/books?id=VxhNuQEACAAJ

32. M. Panwar and P. Singh Mehra. 2011. Hand gesture recognition for human computer interaction. In 2011 International Conference on Image Information Processing. 1-7. DOI :

http://dx.doi.org/10.1109/ICIIP.2011.6108940

33. P. Patel, B. Bhatt, and B. Patel. 2017. Human body posture recognition? A survey. In 2017 International Conference on Innovative Mechanisms for Industry Applications (ICIMIA). 473-477. DOI :

http://dx.doi.org/10.1109/ICIMIA. 2017.7975660

34. Bastian Pfleging, Maurice Rang, and Nora Broy. 2016. Investigating User Needs for Non-driving-related Activities During Automated Driving. In Proc. MUM '16. ACM, New York, NY, USA, 91-99. DOI :

http://dx.doi.org/10.1145/3012709.3012735

35. S. Prabhakar, S. Pankanti, and A. K. Jain. 2003. Biometric recognition: security and privacy concerns. IEEE Security Privacy 1, 2 (March 2003), 33-42. DOI : http://dx.doi.org/10.1109/MSECP. 2003.1193209

36. Andreas Riener, Myounghoon Jeon, Ignacio Alvarez, and Anna K. Frison. 2017. Driver in the Loop: Best Practices in Automotive Sensing and Feedback Mechanisms. Springer International Publishing, Cham, 295-323. DOI :

http://dx.doi.org/10.1007/978-3-319-49448-7_11 
37. Kashif Shaheed, Hangang Liu, Gongping Yang, Imran Qureshi, Jie Gou, and Yilong Yin. 2018. A Systematic Review of Finger Vein Recognition Techniques. Information 9, 9 (2018). DOI :

http://dx.doi.org/10.3390/info9090213

38. Fuming Shih, Ilaria Liccardi, and Daniel Weitzner. 2015. Privacy Tipping Points in Smartphones Privacy Preferences. In Proc. CHI '15. ACM, New York, NY, USA, 807-816. DOI :

http://dx.doi.org/10.1145/2702123.2702404

39. William E. Spangler, Mordechai Gal-Or, and Jerrold H. May. 2003. Using Data Mining to Profile TV Viewers. Commun. ACM 46, 12 (Dec. 2003), 66-72. DOI : http://dx.doi.org/10.1145/953460.953461

40. Jacopo Staiano, Nuria Oliver, Bruno Lepri, Rodrigo de Oliveira, Michele Caraviello, and Nicu Sebe. 2014. Money Walks: A Human-centric Study on the Economics of Personal Mobile Data. In Proc. UbiComp '14. ACM, New York, NY, USA, 583-594. DOI : http://dx.doi.org/10.1145/2632048.2632074

41. Desney Tan and Anton Nijholt. 2010. Brain-Computer Interfaces and Human-Computer Interaction. Springer London, London, 3-19. DOI :

http://dx.doi.org/10.1007/978-1-84996-272-8_1

42. Zhen Tu, Runtong Li, Yong Li, Gang Wang, Di Wu, Pan Hui, Li Su, and Depeng Jin. 2018. Your Apps Give You Away: Distinguishing Mobile Users by Their App Usage Fingerprints. Proc. ACM Interact. Mob. Wearable Ubiquitous Technol. 2, 3, Article 138 (Sept. 2018), 23 pages. DOI : http://dx.doi .org/10.1145/3264948

43. M. Van Ly, S. Martin, and M. M. Trivedi. 2013. Driver classification and driving style recognition using inertial sensors. In 2013 IEEE Intelligent Vehicles Symposium (IV). 1040-1045. DOI :

http://dx.doi.org/10.1109/IVS. 2013.6629603
44. Maria Villa, Mikhail Gofman, and Sinjini Mitra. 2018. Survey of Biometric Techniques for Automotive Applications. In Information Technology - New Generations, Shahram Latifi (Ed.). Springer International Publishing, Cham, 475-481.

45. Jonas Walter and Bettina Abendroth. 2018. Losing a Private Sphere? A Glance on the User Perspective on Privacy in Connected Cars. In Advanced Microsystems for Automotive Applications 2017, Carolin Zachäus, Beate Müller, and Gereon Meyer (Eds.). Springer International Publishing, Cham, 237-247.

46. Gang Wang, Andrew Gallagher, Jiebo Luo, and David Forsyth. 2010. Seeing People in Social Context: Recognizing People and Social Relationships. In Computer Vision - ECCV 2010, Kostas Danilidis, Petros Maragos, and Nikos Paragios (Eds.). Springer Berlin Heidelberg, Berlin, Heidelberg, 169-182.

47. Dustin L Winters, Kevin E Spaulding, and Andrew C Gallagher. 2013. Method of person identification using social connections. (April 9 2013). US Patent $8,416,997$.

48. Jed R. Wood and Larry E. Wood. 2008. Card Sorting: Current Practices and Beyond. J. Usability Studies 4, 1 (Nov. 2008), 1-6. http: //dl.acm.org/citation. cfm?id=2835577. 2835578

49. Yi-Hsuan Yang, Yu-Ching Lin, and Homer Chen. 2009. Personalized Music Emotion Recognition. In Proceedings of the 32Nd International ACM SIGIR Conference on Research and Development in Information Retrieval (SIGIR '09). ACM, New York, NY, USA, 748-749. DOI :

http://dx.doi.org/10.1145/1571941.1572109 